\title{
Determination of the backbone torsion psi angle by tensor correlation of chemical shift anisotropy and heteronuclear dipole-dipole interaction
}

\author{
Yun Mou, Tim W.T. Tsai, Jerry C.C. Chan* \\ Department of Chemistry, National Taiwan University, No. 1, Section 4, Roosevelt Road, Taipei, Taiwan
}

Received 28 June 2006; received in revised form 25 December 2006

Dedicated to Professor Sunney I. Chan on the occasion of his 70 th birthday

Available online 26 January 2007

\begin{abstract}
We demonstrate that the backbone torsion psi angle of a uniformly labeled residue can be determined accurately by correlating the chemical shift anisotropy of the carbonyl carbon and the ${ }^{13} \mathrm{C}-{ }^{1} \mathrm{H}$ heteronuclear dipole-dipole interaction of the alpha carbon. To obtain the highest sensitivity for the psi angle determination, the following conditions are desired: (i) the recoupling pulse sequences for the CSA and the heteronuclear dipolar interactions are gamma encoded, in which the spatial parts of $m=2$ are selected; (ii) the homonuclear polarization transfer is based on the scalar spin-spin coupling. Experimental data were obtained for $\left[\mathrm{U}-{ }^{13} \mathrm{C},{ }^{15} \mathrm{~N}\right]$-alanine and $\mathrm{N}$-acetyl$\left[\mathrm{U}-{ }^{13} \mathrm{C},{ }^{15} \mathrm{~N}\right]-\mathrm{D}, \mathrm{L}-\mathrm{valine}$ under magic-angle spinning at $25 \mathrm{kHz}$. Only three data points are required for the measurements and the dihedral angles determined are in excellent agreement with the diffraction data.
\end{abstract}

(C) 2007 Elsevier Inc. All rights reserved.

Keywords: RACO; CSA; MAS; Gamma-encoded

\section{Introduction}

Solid state nuclear magnetic resonance (SSNMR) is a powerful technique for the structural elucidation of biological solids [1-3]. There are many different SSNMR techniques suggested for the determination of peptide backbone torsion angles $\phi$ and $\psi$ under magic-angle spinning (MAS) [4-8]. These techniques are all based on the principle of tensor correlation. Most techniques for $\psi$ determination require isotopic labeling of two consecutive residues [9-16]. Nevertheless, it has been demonstrated by Ishii et al. that the backbone torsion $\psi$ angle can be determined by correlating the carbonyl ${ }^{13} \mathrm{C}$ chemical shift anisotropy (CSA) and the ${ }^{13} \mathrm{C}_{\alpha-}{ }^{1} \mathrm{H}_{\alpha}$ dipolar tensors within a single ${ }^{13} \mathrm{C}$ uniformly labeled residue [17]. This approach, with the acronym RACO for relayed anisotropy correlation, comprises three basic modules, viz. the CSA recoupling of $\mathrm{C}^{\prime}$, the polarization transfer from $\mathrm{C}^{\prime}$ to $\mathrm{C}_{\alpha}$, and the heteronuclear ${ }^{13} \mathrm{C}_{\alpha}-{ }^{1} \mathrm{H}_{\alpha}$ dipolar recoupling [17]. In its original implementation, RACO requires the acquisition

\footnotetext{
${ }^{*}$ Corresponding author. Fax: +886223636359 .

E-mail address: chanjcc@ntu.edu.tw (J.C.C. Chan).
}

of a series of $2 \mathrm{D}$ spectra under the heteronuclear dipolar recoupling condition and therefore is not applicable for systems with multiple uniformly ${ }^{13} \mathrm{C}$ labeled residues. This limitation of RACO can be easily alleviated by separating the heteronuclear dipolar recoupling and the $t_{2}$ acquisition periods, and by choosing recoupling pulse sequences designed for fast MAS [18]. However, it is not trivial to obtain the optimized implementation of RACO because there are many different recoupling pulse sequences reported in the literature [19]. In this work, we present a simple physical picture to illustrate the underlying spin dynamics of the RACO approach, from which it can be shown that the following conditions are desired for the optimized implementation of RACO: (i) the recoupling pulse sequences for the CSA and the heteronuclear dipolar interactions are gamma encoded, in which the spatial parts of $m=2$ are selected; (ii) the homonuclear polarization transfer is based on the scalar spin-spin coupling. Experimental data were obtained for $\left[\mathrm{U}-{ }^{13} \mathrm{C},{ }^{15} \mathrm{~N}\right]$-alanine and $\mathrm{N}$-acetyl-[U- $\left.{ }^{13} \mathrm{C},{ }^{15} \mathrm{~N}\right]$-D,L-valine under $\mathrm{MAS}$ at $25 \mathrm{kHz}$. Only three data points are required for the measurements and the dihedral angles determined are in excellent agreement with the diffraction data. 


\section{Theory}

\subsection{Gamma-encoded Hamiltonians}

To facilitate our discussion, we will write down the analytical expressions of the so-called $\gamma$-encoded average Hamiltonians for the CSA and the heteronuclear dipolar recouplings [20]. In general, the Hamiltonian of an NMR interaction can be written as a product of the spatial and the spin tensors [21]. Under MAS at frequency $\omega_{r}=2 \pi v_{r}$, the high-field Hamiltonian of an internal interaction with its spatial part described by a second-rank tensor takes the following form in the rotating frame [22]:

$H(t)=H_{\mathrm{rf}}(t)+H_{\text {int }}(t)$,

$H_{\mathrm{int}}(t)=\sum_{\Lambda m \lambda \mu} \omega_{m}^{\Lambda} \exp \left(\mathrm{im} \omega_{r} t\right) T_{\lambda 0}^{\Lambda}$

where $H_{\text {int }}(t)$ will periodically oscillate with two frequencies, viz. $\omega_{r}(m=1)$ and $2 \omega_{r}(m=2)$. On the basis of the pulse symmetry developed by Levitt et al. [20], one could selectively recouple a specific spatial part with $m$ equal to 1 and/or 2 for the average Hamiltonian calculated to the lowest order. While the magnitude of an average Hamiltonian is in general dependent on all the three Euler angles $(\alpha, \beta, \gamma)$, a pulse sequence is known as $\gamma$ encoding if the magnitude of the corresponding average Hamiltonian is independent of the third Euler angle $\gamma$ [20]. In particular, the $\gamma$-encoded Hamiltonian for the CSA can be written as $[23,24]$

$$
\begin{aligned}
\bar{H}_{0}^{\mathrm{CSA}}= & c^{\mathrm{CS}} C_{m} \chi_{m}\left\{\exp \left[-\mathrm{i}\left(m \gamma-\delta_{m}-\varepsilon\right)\right] S_{ \pm}\right. \\
& \left.+\exp \left[\mathrm{i}\left(m \gamma-\delta_{m}-\varepsilon\right)\right] S_{\mp}\right\},
\end{aligned}
$$

$$
\begin{aligned}
C_{1}(\alpha, \beta) & \\
= & \frac{\left(\delta_{33}-\delta_{\text {iso }}\right)}{6 \sqrt{2}} \\
& \times \sqrt{(3 \sin 2 \beta-\eta \sin 2 \beta \cos 2 \alpha)^{2}+(2 \eta \sin \beta \sin 2 \alpha)^{2}},
\end{aligned}
$$

$$
\begin{aligned}
& C_{2}(\alpha, \beta) \\
& \quad=\frac{\left(\delta_{33}-\delta_{\text {iso }}\right)}{12} \\
& \quad \times \sqrt{\left(3 \sin ^{2} \beta+\eta\left(1+\cos ^{2} \beta\right) \cos 2 \alpha\right)^{2}+(2 \eta \cos \beta \sin 2 \alpha)^{2}},
\end{aligned}
$$

$\eta=\frac{\delta_{11}-\delta_{22}}{\delta_{33}-\delta_{\text {iso }}}$

where $\delta_{11}, \delta_{22}$ and $\delta_{33}$ are the principal components of the $\mathrm{CS}$ tensor; $c^{\mathrm{CS}}$ is a collection of physical constants; $\alpha, \beta$ and $\gamma$ are the Euler angles bringing the principal axis system (PAS) of the chemical shift tensor to the rotor-fixed coordinate; $\chi_{m}$ denotes the scaling factor of the pulse sequence [25]; other symbols in the brace brackets carry their meanings defined by Terao and co-workers [23]. In the toggling frame transformed by a $\pi / 2$ pulse of the $S$ channel, the average Hamiltonian of the heteronuclear dipole-dipole interaction due to a $\gamma$-encoded pulse sequence can be expressed as follows:

$$
\begin{aligned}
\bar{H}_{0}^{D_{\mathrm{IS}}}= & c^{D_{\mathrm{IS}}} C_{m} \chi_{m}\left\{\exp [-\mathrm{i}(m \gamma-\varepsilon)] S_{x} I_{\mp}\right. \\
& \left.+\exp [\mathrm{i}(m \gamma-\varepsilon)] S_{x} I_{ \pm}\right\} \\
C_{1}\left(\beta^{\prime}\right)= & \frac{1}{2 \sqrt{2} r_{\mathrm{IS}}^{3}} \sin 2 \beta^{\prime}, \\
C_{2}\left(\beta^{\prime}\right)= & \frac{1}{4 r_{\mathrm{IS}}^{3}} \sin ^{2} \beta^{\prime}, \\
\cos \beta^{\prime}= & \left(\cos \left(\psi-30^{\circ}\right) \sin { }^{2} \beta+\cos { }^{2} \beta\right) \cos \left(\beta-108.9^{\circ}\right) \\
& \left.-\left(-1+\cos \left(\psi-30^{\circ}\right)\right) \sin \beta \cos \beta \sin \left(\beta-108.9^{\circ}\right)\right] \\
& \times[-\sin \beta \cos \alpha \cos \beta+\sin \beta \cos \beta] \\
& +\left[-\sin \left(\psi-30^{\circ}\right) \sin \beta \cos \left(\beta-108.9^{\circ}\right)\right. \\
& \left.+\sin \left(\psi-30^{\circ}\right) \cos \beta \sin \left(\beta-108.9^{\circ}\right)\right] \\
& \times[\sin \alpha \sin \beta]+\left[\left(-1+\cos \left(\psi-30^{\circ}\right)\right) \sin ^{\circ}\right. \\
& \times \cos \beta \cos \left(\beta-108.9^{\circ}\right)-\left(\cos \left(\psi-30^{\circ}\right) \cos ^{2} \beta\right. \\
& \left.\left.+\sin { }^{2} \beta\right) \sin \left(\beta-108.9^{\circ}\right)\right]\left[\cos \alpha \sin ^{2} \beta+\cos ^{2} \beta\right],
\end{aligned}
$$

where $\psi$ is the backbone torsion angle to be determined and the PAS of the chemical shift tensor is taken as our reference frame (Fig. 1). Consequently, the absolute magnitudes of both the $\gamma$-encoded average Hamiltonians for the CSA and the heteronuclear dipole-dipole interactions have the following generic form:

$\bar{\omega}_{m}^{\Lambda}=\left|c^{\Lambda} C_{m} \chi_{m}\right|$.

Eq. (10) is the major result of this section, which allows us to calculate the magnitudes of the $\gamma$-encoded average Hamiltonians for the CSA $\left(\bar{\omega}_{m}^{\mathrm{CS}}\right)$ and the heteronuclear dipole-dipole $\left(\bar{\omega}_{m}^{D_{\mathrm{IS}}}\right)$ interactions for a given backbone torsion $\psi$ angle, on the basis of Eq. (11).

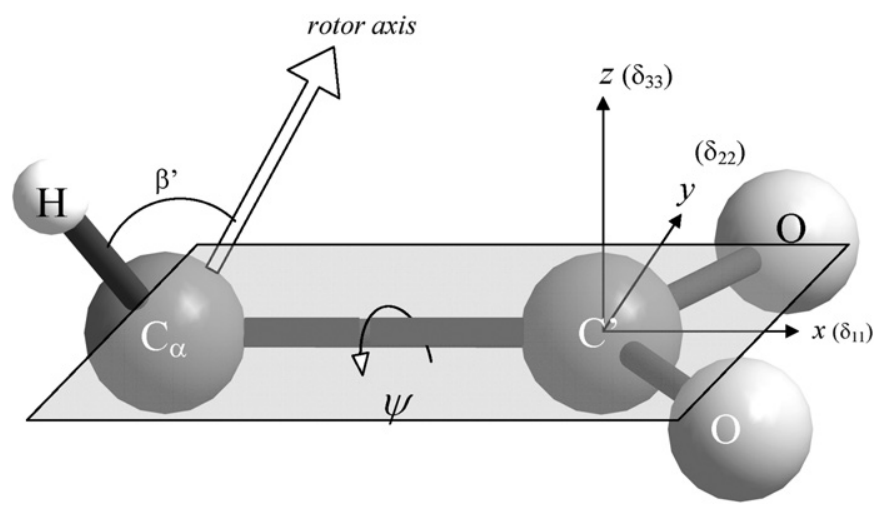

Fig. 1. Illustration of the reference frame employed in our calculations involving Eqs. (3)-(15) 


\subsection{Correlation of $\bar{\omega}_{m}^{\mathrm{CS}}$ and $\bar{\omega}_{m}^{D_{\mathrm{IS}}}$}

Because the RACO experiment shows that one can determine the $\psi$ angle by correlating the CSA and the heteronuclear dipole-dipole interactions, $\bar{\omega}_{m}^{\mathrm{CS}}$ and $\bar{\omega}_{m}^{D_{\mathrm{IS}}}$ should be correlated to a certain extent. To verify this physical intuition, we calculated $\bar{\omega}_{m}^{\mathrm{CS}}$ and $\bar{\omega}_{m}^{D_{\text {IS }}}$ based on the geometrical [26] and NMR parameters [27] of L-alanine. The orientation of the chemical shift tensor with respect to the molecule was taken from the single-crystal NMR data reported for L-alanine [27], except that the orientation of the $\delta_{11}$ component of the $\mathrm{C}^{\prime}$ CSA tensor was set to bisect the $\mathrm{O}-\mathrm{C}-\mathrm{O}$ angle of the carboxylate group. On the basis of Eqs. (3)-(11), the magnitudes of $\bar{\omega}_{m}^{\mathrm{CS}}$ and $\bar{\omega}_{m}^{D_{\mathrm{IS}}}$ were calculated for $\gamma$-encoded Hamiltonians. A total of 700 REPULSION angles ( $\alpha$ and $\beta$ ) was applied to sample the crystallite orientations [28]. To reveal any correlation of $\bar{\omega}_{m}^{\mathrm{CS}}$ and $\bar{\omega}_{m}^{D_{\mathrm{IS}}}$, we performed a linear regression analysis of the plot of $\bar{\omega}_{m}^{\mathrm{CS}}$ versus $\bar{\omega}_{m}^{D_{\text {IS }}}$. Clearly, there are four possible plots for $\gamma$-encoded Hamiltonians, viz. $\bar{\omega}_{m=1}^{\mathrm{CS}} \leftrightarrow \bar{\omega}_{m=1}^{D_{\mathrm{IS}}}$, $\bar{\omega}_{m=1}^{\mathrm{CS}} \leftrightarrow \bar{\omega}_{m=2}^{D_{\mathrm{IS}}}, \quad \bar{\omega}_{m=2}^{\mathrm{CS}} \leftrightarrow \bar{\omega}_{m=1}^{D_{\mathrm{IS}}} \quad$ and $\quad \bar{\omega}_{m=2}^{\mathrm{CS}} \leftrightarrow \bar{\omega}_{m=2}^{D_{\mathrm{IS}}}$. Fig. 2 illustrates the calculated results for $\psi$ equal to $30^{\circ}$ and $120^{\circ}$, showing that the slopes of the regression lines and the correlation coefficients depend on the $\psi$ angle. For clarity, the results calculated for other $\psi$ angles are not shown. Overall, we find that the regression lines of $\bar{\omega}_{m=2}^{\mathrm{CS}} \leftrightarrow \bar{\omega}_{m=2}^{D_{\mathrm{IS}}}$ give the highest correlation coefficients and the correlation coefficients of other $\bar{\omega}_{m}^{\mathrm{CS}} \leftrightarrow \bar{\omega}_{m}^{D_{\text {IS }}}$ plots are considerably lower.

There are three simple physical scenarios that one may envision in Fig. 2: (i) the spin packets which evolve rapidly (slowly) under the CSA interaction evolve again rapidly (slowly) during the dipolar recoupling period; (ii) the spin packets which evolve rapidly (slowly) under the CSA interaction evolve slowly (rapidly) during the dipolar recoupling period; and (iii) the evolution of the spin packets does not have any correlation during the two recoupling periods. For convenience, these three scenarios are named as positive correlation, negative correlation, and no correlation, respectively. Referring to Fig. 2, the plot of $\bar{\omega}_{m=2}^{\mathrm{CS}} \leftrightarrow \bar{\omega}_{m=2}^{D_{\text {IS }}}$ has positive and negative correlations for $\psi$ equal to $30^{\circ}$ and $120^{\circ}$, respectively. Although the discussion in this section may appear qualitative, it provides the necessary foundation for our subsequent discussion that $\bar{\omega}_{m=2}^{\mathrm{CS}}$ and $\bar{\omega}_{m=2}^{D_{\mathrm{IS}}}$ do have correlations for certain $\psi$ angles. Before we trace the connection between the correlation of $\bar{\omega}_{m=2}^{\mathrm{CS}} \leftrightarrow \bar{\omega}_{m=2}^{D_{\text {IS }}}$ and the $\psi$ angle determination, we will first discuss the "echo" phenomenon associated with the RACO experiment.

\subsection{Echo of polarization}

The following discussion will be based on the framework of RACO. To reiterate, the RACO experiment comprises the CSA recoupling of $\mathrm{C}^{\prime}$, the polarization transfer from $\mathrm{C}^{\prime}$ to $\mathrm{C}_{\alpha}$, and the heteronuclear ${ }^{13} \mathrm{C}_{\alpha}{ }^{-1} \mathrm{H}_{\alpha}$ dipolar recoupling [17]. We choose to create an isotropic zero-quantum Hamiltonian for the homonuclear polarization transfer [29-36]. Assume that the system is initially at thermal equilibrium. Under the influence of the above $\gamma$-encoded chemical shift Hamiltonian (Eq. (3)), the density operator calculated at each crystallite orientation $(\alpha, \beta, \gamma)$ is generally written as

$$
\rho_{\alpha, \beta, \gamma}(t)=a_{x}(t) S_{x}+a_{y}(t) S_{y}+a_{z}(t) S_{z} .
$$

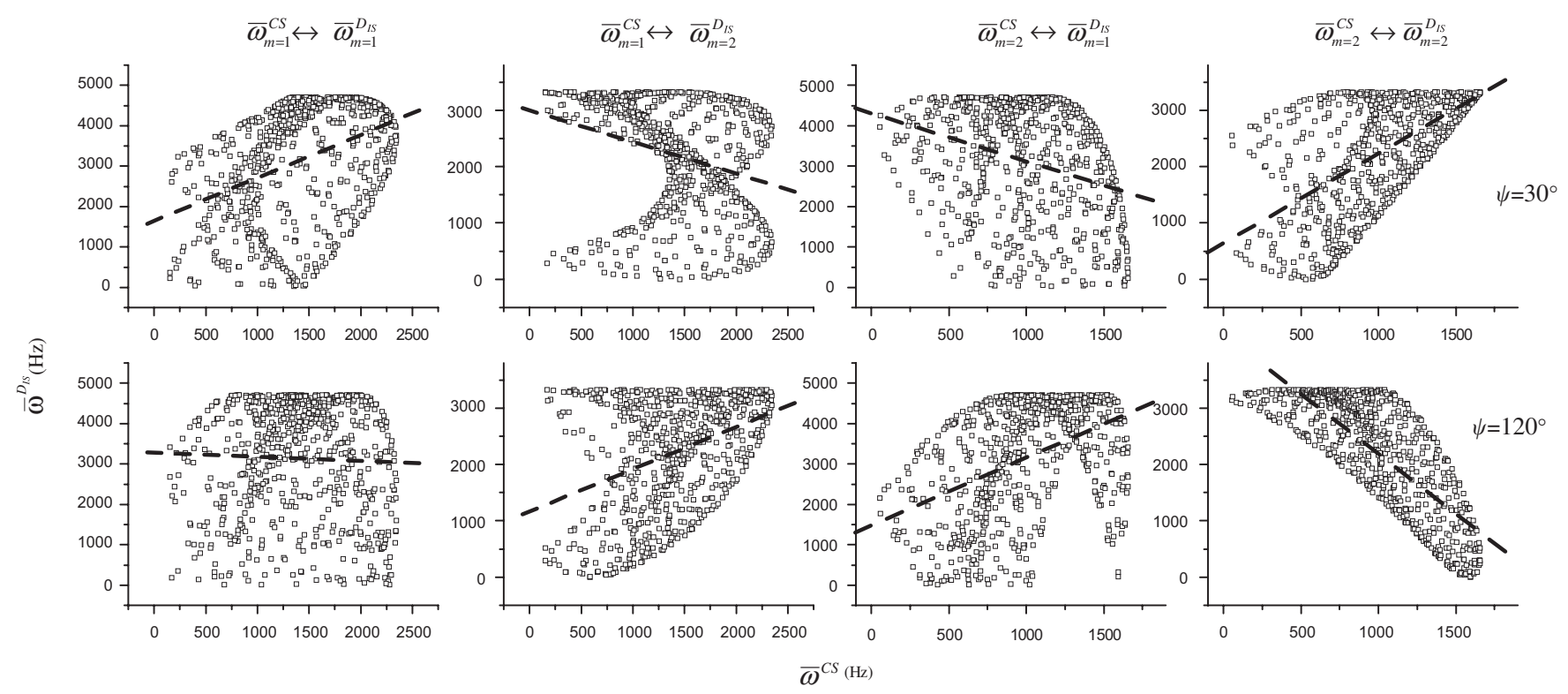

Fig. 2. Plot of $\bar{\omega}_{m}^{\mathrm{CS}}$ versus $\bar{\omega}_{m}^{D_{\mathrm{IS}}}$ for $\psi$ equal to $30^{\circ}$ and $120^{\circ}$. The dashed lines represent the regression lines obtained by linear regression analyses. The regression coefficients of $\bar{\omega}_{m=2}^{\mathrm{CS}} \leftrightarrow \bar{\omega}_{m=2}^{D_{\mathrm{IS}}}$ were found to be 0.59 and -0.79 for $\psi$ equal to $30^{\circ}$ and $120^{\circ}$, respectively. 
Due to the symmetry property of the $\gamma$-encoded Hamiltonian, after powder averaging the spin system can be simply calculated as

$\rho(t)=\left\langle a_{z}(t) S_{z}\right\rangle$,

$a_{z}(t)=\cos \left(\bar{\omega}_{m=2}^{\mathrm{CS}} t\right)$,

where the bracket $\langle\cdots\rangle$ denotes powder averaging; $\bar{\omega}_{m=2}^{\mathrm{CS}}$ is given by Eqs. (3)-(6) and (11). As will become apparent shortly, it proves useful to decompose $\rho(t)$ into the positive and negative $S_{z}$ components, viz. $\rho_{+z}(t)$ and $\rho_{-z}(t)$. Each of these components is just the vector sum of $\rho_{\alpha, \beta, \gamma}(t)$ whose $a_{z}(t)$ are of the same sign. That is, we have $\rho(t)=\rho_{+z}$ $(t)+\rho_{-z}(t)$. The trajectories of $\rho(t), \rho_{+z}(t)$ and $\rho_{-z}(t)$ during the CSA recoupling period were calculated on the basis of Eq. (3), in which $\bar{\omega}_{m=2}^{\mathrm{CS}}$ was selected. At the first glance, one has to choose a particular pulse sequence for the calculation of the trajectories because different pulse sequences may have different scaling factors, i.e. $\chi_{m}$ of Eq. (3). To make the calculated results independent of $\chi_{m}$, we choose to present our results in a relative time scale. That is, we arbitrarily define the time unit $(T)$ as the time required for the fastest evolving spin packet to advance a phase angle of $\pi / 2$. Note that in the absolute time scale, the larger the factor $\chi_{m}$, the smaller the value of $T$. The calculated trajectories of $\rho(t), \rho_{+z}(t)$ and $\rho_{-z}(t)$ are shown in Fig. 3. Consider the spin system at $t=1.5 T$, the positive and negative components highlighted in Fig. 3 are of similar magnitudes and therefore the polarization along the $z$-axis is very small. We then choose to transfer this highlighted longitudinal spin order to the alpha carbon based on an isotropic zero-quantum Hamiltonian. Subsequently, we recouple the $\mathrm{C}_{\alpha}-\mathrm{H}_{\alpha}$ heteronuclear dipolar

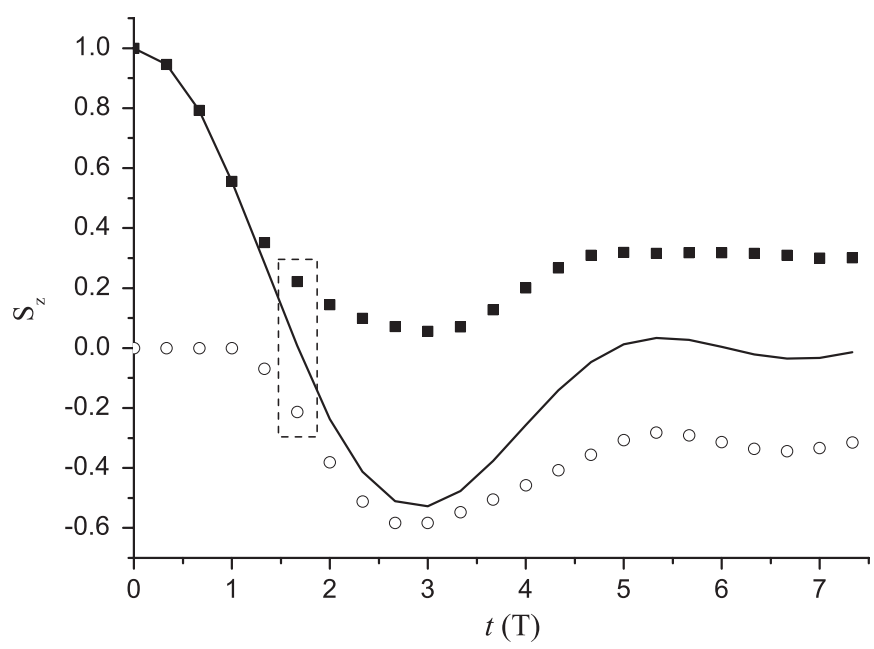

Fig. 3. Decomposition of the density operator into the positive (filled squares) and negative (open circles) components, viz. $\rho_{+z}(t)$ and $\rho_{-z}(t)$ evolving under CSA recoupling. The time axis has a relative scale, in which the unit $(T)$ is set to the time required for the fastest evolving spin packet to advance a phase angle of $\pi / 2$. The positive and negative components highlighted in the rectangular box are, respectively, referred to as the slow- and the fast-evolving components in the text. interaction. The average Hamiltonian is again $\gamma$ encoded and the term $\bar{\omega}_{m=2}^{D_{\text {IS }}}$ is selected (see Eq. (7)). The density operator is then calculated as

$\rho(t)=S_{z}\left\langle\cos \left(\bar{\omega}_{m=2}^{\mathrm{CS}} \tau_{\mathrm{CSA}}\right) \cos \left(\bar{\omega}_{m=2}^{D_{\mathrm{IS}}} t\right)\right\rangle$,

where $\tau_{\mathrm{CSA}}$ denotes the evolution time during the CSA recoupling period, i.e. $\tau_{\mathrm{CSA}}=1.5 T$. As shown in Fig. 4 , the density operator $\rho(t)$ during the dipolar recoupling period was calculated for $\psi$ equal to $30^{\circ}$ and $120^{\circ}$, in which the polarization along the $z$-axis has a positive or negative "echo", respectively. This phenomenon is a reminiscence of the classical spin-echo concept. Roughly speaking, the polarization was "dephased" during the CSA recoupling period and "refocused" during the dipolar recoupling period. In the following section, the evolution of the
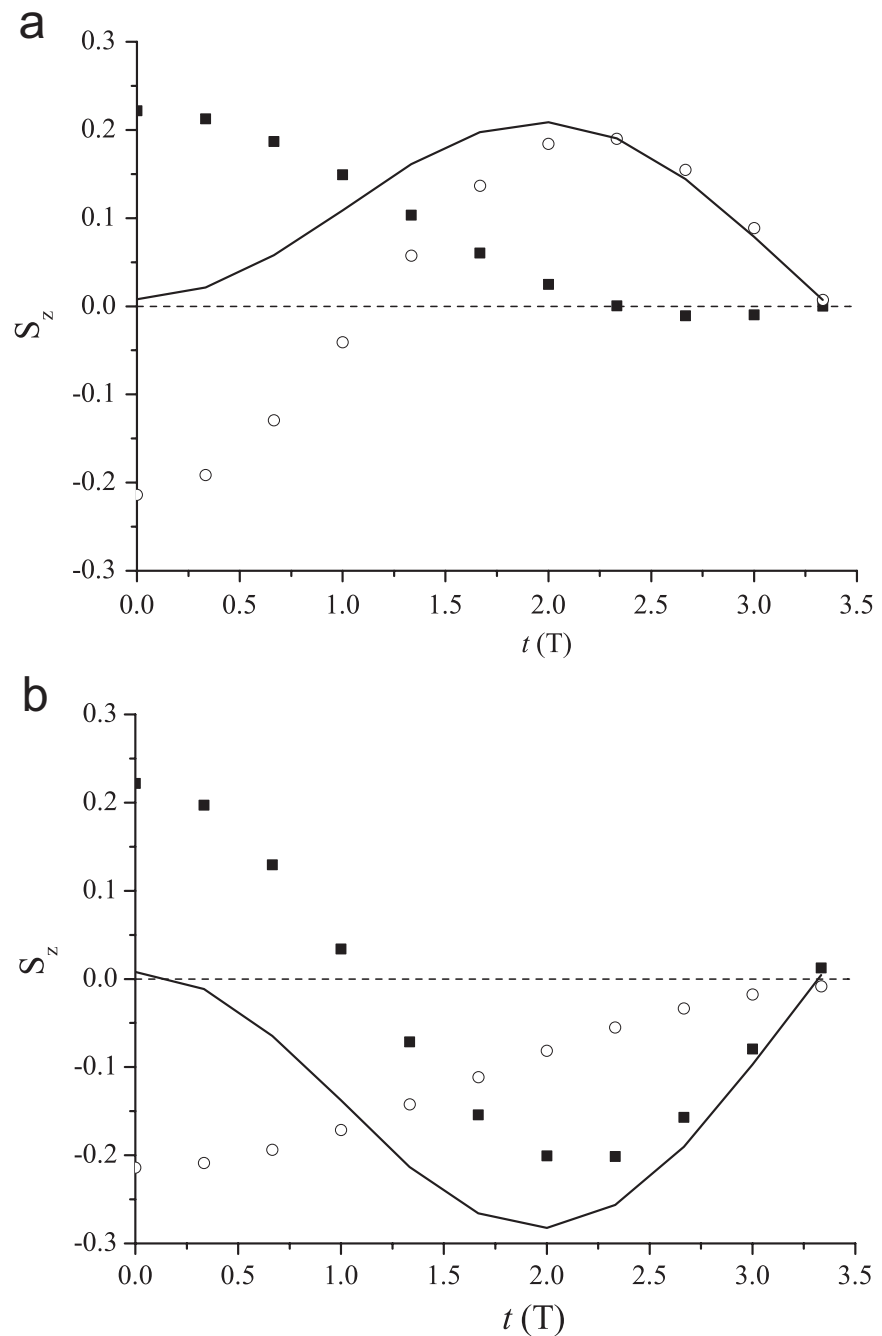

Fig. 4. Evolution of the spin system during the $\mathrm{C}_{\alpha}-\mathrm{H}_{\alpha}$ heteronuclear dipolar recoupling period. The initial state corresponds to the longitudinal spin order highlighted in Fig 3. The solid lines denote the evolution of the density operator calculated on the basis of Eq. (15). The filled squares and open circles describe the trajectories of the slow- and fast-evolving components, respectively. (a) $\psi=30^{\circ}$ and (b) $\psi=120^{\circ}$. Other conditions are identical to those described in Fig. 3. 


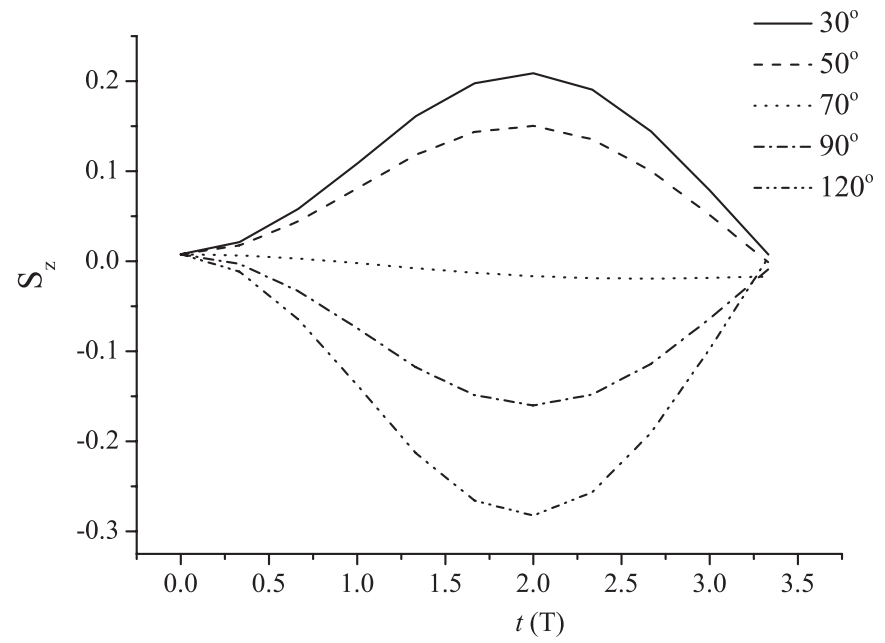

Fig. 5. Evolution of the spin system during the $\mathrm{C}_{\alpha}-\mathrm{H}_{\alpha}$ heteronuclear dipolar recoupling period calculated for different $\psi$ angles. Because of the degeneracy (shown in Fig. 9), only the results calculated for $30 \leqslant \psi \leqslant 120$ are shown. The lines denote the evolution of the density operator after powder averaging. Other conditions are identical to those described in Figs. 3 and 4. longitudinal spin order created by CSA recoupling is followed explicitly during the dipolar recoupling period so that the underlying spin dynamics of the polarization echo formation can be better appreciated.

\subsection{Optimized RACO experiment}

Referring to Fig. 3, the components $\rho_{-z}(t)$ and $\rho_{+z}(t)$ at $t=1.5 T$ are identified with the fast- and slow-evolving spin packets, respectively. Although the terms fast- and slowevolving are rather subjective and arbitrary, they serve as two convenient descriptors for the subsequent discussion. Basically, these two descriptors divide all the orientations of our powder averaging scheme into two subsets. Each of these subsets corresponds to the orientations contributing to the fast- or the slow-evolving components during the CSA recoupling period. Accordingly, the filled squares and open circles in Fig. 4 illustrate the evolution trajectories of these slow- and fast-evolving components, respectively, during the $\mathrm{C}_{\alpha}-\mathrm{H}_{\alpha}$ heteronuclear dipolar recoupling period. Note that the addition of these two components will give

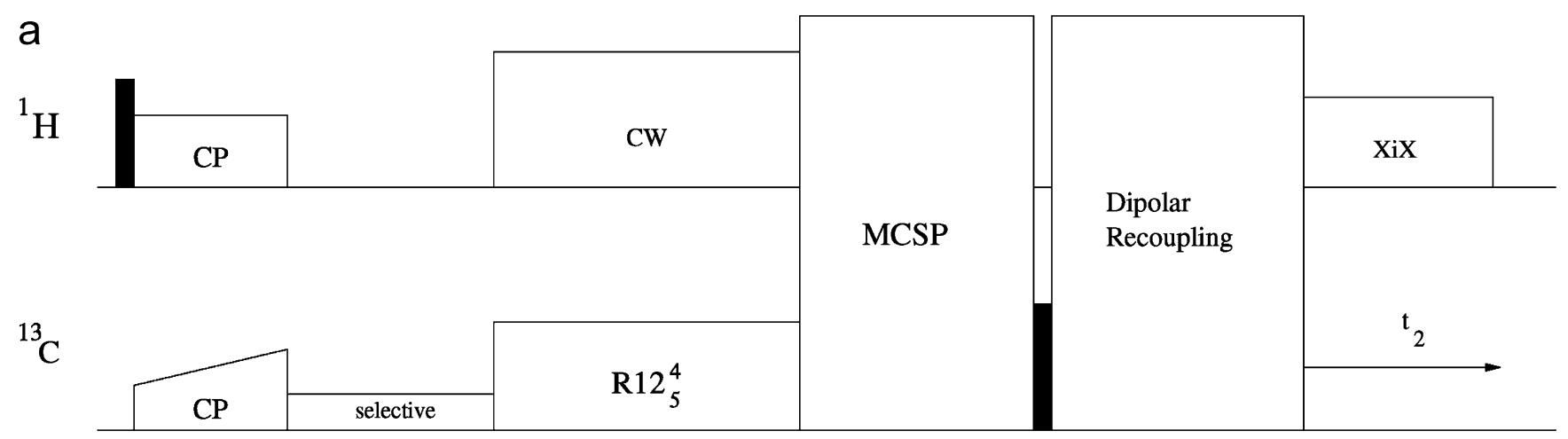

b
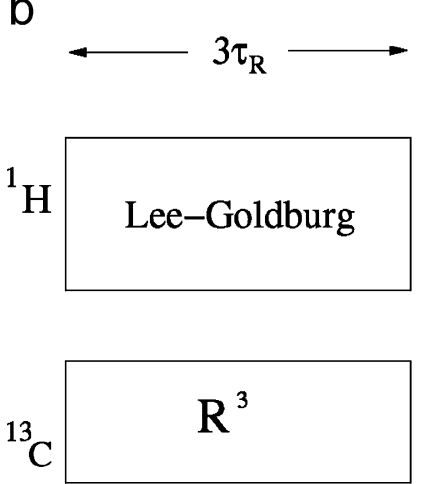

C

${ }^{1} \mathrm{H}$

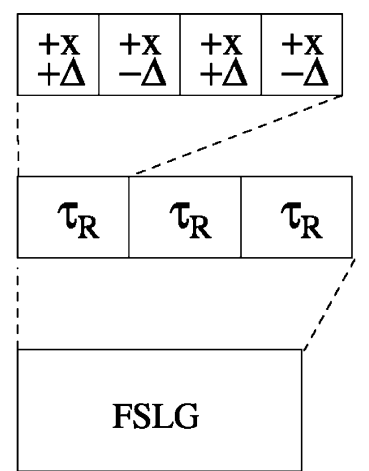

Fig. 6. (a) Pulse sequence for the determination of the backbone torsion $\psi$ angle. The selective module for the $\mathrm{C}^{\prime}$ signal contains a hard $\pi / 2$ pulse followed by a Gaussian $\pi / 2$ pulse (placed in the aliphatic region) and a $z$-filter. Two pulse sequences for the dipolar recoupling are tested: (b) gamma-encoded dipolar recoupling sequence, rotary resonance; (c) non- $\gamma$-encoded dipolar recoupling sequence, FSLG $(m=2)$. 
the density operators denoted by the solid lines. We have shown earlier that $\bar{\omega}_{m=2}^{\mathrm{CS}}$ and $\bar{\omega}_{m=2}^{D_{\mathrm{IS}}}$ have a positive correlation for $\psi$ equal to $30^{\circ}$. Consequently, there is a positive polarization echo observed in Fig. 4(a) because the slow-evolving (fast-evolving) component of the CSA recoupling period evolves again slowly (rapidly) in the dipolar recoupling period. That is, a positive "echo" is formed when the two components align in the same direction. The negative "echo" shown in Fig. 4(b) is similarly rationalized by the fact that $\bar{\omega}_{m=2}^{\mathrm{CS}}$ and $\bar{\omega}_{m=2}^{D_{\mathrm{IS}}}$ have a negative correlation for $\psi$ equal to $120^{\circ}$. It is noteworthy that the higher the correlation coefficient of $\bar{\omega}_{m=2}^{\mathrm{CS}}$ and $\bar{\omega}_{m=2}^{D_{\mathrm{IS}}}$, the larger the "echo".

The foregoing discussion has an important implication in the determination of the backbone torsion $\psi$ angle. It can be surmised that $\bar{\omega}_{m=2}^{\mathrm{CS}}$ and $\bar{\omega}_{m=2}^{D_{\mathrm{IS}}}$ can have a positive correlation, a negative correlation or no correlation, depending on the $\psi$ value. As a result, the signal intensity of the "echo" can serve as a sensitive measure for the $\psi$ angle. To illustrate this point, we show the calculated density operator $\rho(t)$ during the dipolar recoupling period for different $\psi$ angles in Fig. 5. Clearly, the span of the echo intensities depends on the intensities of the most positive and the most negative "echoes". We can now conclude that the $\gamma$-encoding pulse sequence selecting spatial part with $m$ equal to 2 are optimized for RACOlike experiments because $\bar{\omega}_{m=2}^{\mathrm{CS}}$ and $\bar{\omega}_{m=2}^{D_{\mathrm{IS}}}$ have the most positive and the most negative correlation coefficients (Fig. 2).

Up to the present stage, we have been focusing on the correlation between two $\gamma$-encoded recoupling periods. Interestingly, additional simulations show that the span of the "echo" intensities relevant to $\psi$ angle determination is only slightly diminished when we employ a non- $\gamma$-encoded pulse sequence for the dipolar recoupling period (vide infra). This finding is very useful for practical consideration because it is not trivial to find a suitable $\gamma$-encoded pulse sequence which can recouple the ${ }^{13} \mathrm{C}-{ }^{1} \mathrm{H}$ dipole-dipole interaction and decouple the ${ }^{1} \mathrm{H}-{ }^{1} \mathrm{H}$ dipole-dipole interaction simultaneously.

\section{Experimental methods}

Uniformly ${ }^{13} \mathrm{C}$ - and ${ }^{15} \mathrm{~N}$-labeled L-alanine and L-valine were used as received from Cambridge Isotopes Laboratories. Labeled $N$-acetyl-D,L-valine (NAV) was synthesized by dissolving equal portions $(96 \mathrm{mg}$ ) of unlabeled D-valine and uniformly labeled L-valine in acetic acid $(20 \mathrm{ml})$. The mixture was refluxed for $10 \mathrm{~min}$ in the presence of unlabeled acetic anhydride $(0.26 \mathrm{ml})$. The product was obtained after solvent evaporation under reduced pressure at $85^{\circ} \mathrm{C}$.

All NMR experiments were carried out at ${ }^{13} \mathrm{C}$ and ${ }^{1} \mathrm{H}$ frequencies of 75.5 and $300.1 \mathrm{MHz}$, respectively, on a Bruker DSX300 NMR spectrometer equipped with a commercial $2.5 \mathrm{~mm}$ probe. The spectra were measured at room temperature and at a spinning frequency of $25 \mathrm{kHz}$.
The variation of MAS frequency was limited to $\pm 5 \mathrm{~Hz}$ using a commercial pneumatic control unit. The samples were confined in the middle one-half of the rotor volume using Teflon spacers. During the contact time $(1.5 \mathrm{~ms})$, the ${ }^{1} \mathrm{H}$ nutation frequency was set to $50 \mathrm{kHz}$ and that of ${ }^{13} \mathrm{C}$ was ramped through the HartmannHahn matching condition adiabatically [37,38]. The $\pi / 2$ Gaussian selective pulse was set to $475 \mu$ s long and positioned at the aliphatic region. Recycle delay was set to $2 \mathrm{~s}$. Continuous-wave and $\mathrm{XiX}$ [39] proton decouplings $(100 \mathrm{kHz})$ were applied during the CSA recoupling period and the $t_{2}$ acquisition, respectively. The ${ }^{13} \mathrm{C}$ nutation frequencies were set to 60 and $125 \mathrm{kHz}$ for $R 12_{5}^{4}$ and for the R-TOBSY pulse block, respectively, as required by the pulse symmetries [20,31]. The R-TOBSY mixing time was set to $7.68 \mathrm{~ms}$ for alanine and $5.76 \mathrm{~ms}$ for NAV. The Lee-Goldburg (LG) irradiation was achieved by setting the ${ }^{1} \mathrm{H}$ decoupling field and the resonance offset at 81.6 and $57.8 \mathrm{kHz}$, respectively, so that the
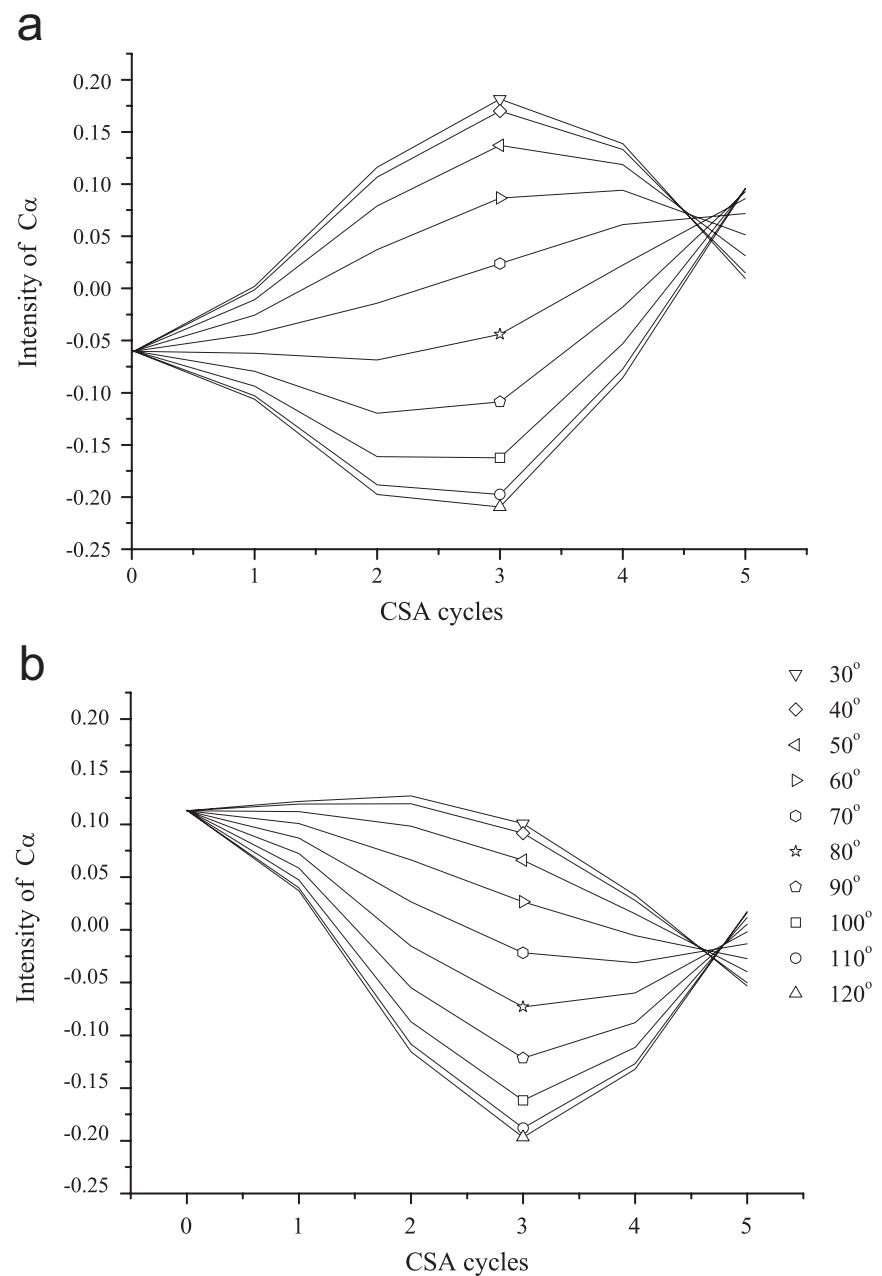

Fig. 7. Calculated $\mathrm{C}_{\alpha}$ signal intensities based on the pulse sequence shown in Fig. 6. The dipolar recoupling period was fixed to three rotor cycles, during which (a) the $\gamma$-encoded rotary resonance and (b) the non- $\gamma$ encoded FSLG $(m=2)$ sequences were employed. 
effective nutation frequency was equal to $100 \mathrm{kHz}$ [40]. The duration of the LG irradiation was fixed to three rotor periods. For each spectrum of L-alanine (NAV), a total of 16 (512) transients was accumulated. Each experimental data point was collected by integrating the corresponding $\mathrm{C}_{\alpha}$ peak area.

Numerical simulations used to compare with experimental data were carried out using SIMPSON (version 1.1.0) [41]. For our simulations, the maximum time step over which the Hamiltonian is approximated to be timeindependent was set to $1 \mu \mathrm{s}$. Typically, a powder averaging scheme containing 100 REPULSION angles $(\alpha$ and $\beta)$ and $18 \gamma$ angles was chosen [28]. Relaxation effects were ignored. Our calculations were done on a three-spin $\left(\mathrm{C}^{\prime}\right.$, $\mathrm{C}_{\alpha}, \mathrm{H}_{\alpha}$ ) system at 7.05T. The ${ }^{13} \mathrm{C}^{\prime} \mathrm{CSA}$ tensors and all other structural parameters of L-alanine [26] and NAV [42] were obtained from the literature. All the pulse durations and the rf fields were matched to the experimental conditions. Simulations were done for the $\psi$ angle varied from $0^{\circ}$ to $180^{\circ}$ at steps of $5^{\circ}$.

\section{Results}

\subsection{Numerical simulations}

We have shown that the approach of $\bar{\omega}_{m=2}^{\mathrm{CS}} \leftrightarrow \bar{\omega}_{m=2}^{D_{\mathrm{IS}}}$ is the most promising one for $\psi$ angle determination. Thus, we proposed to use the pulse sequence shown in Fig. 6 for $\psi$ angle determination. We chose the $R 12_{5}^{4}$ pulse sequence for the CSA recoupling, in which the spin part of the average Hamiltonian comprises the $S_{ \pm}$operators and therefore the initial magnetization should be prepared along the $z$-axis. As required by the pulse symmetry of $R 12_{5}^{4}$, the spatial part of the average Hamiltonian contains the term $\bar{\omega}_{m=2}^{\mathrm{CS}}$ only [20]. The $R 12_{5}^{4}$ pulse sequence with the composite pulse $(90)_{\phi}(270)_{\phi+180}$ incorporated in the basic $R$ elements is very robust with respect to $\mathrm{rf}$ inhomogeneity. The multiple chemical shift precession (MCSP) approach was chosen for the selective polarization transfer from $\mathrm{C}^{\prime}$ to $\mathrm{C}_{\alpha}$ [36]. For the ${ }^{13} \mathrm{C}-{ }^{1} \mathrm{H}$ dipolar recoupling, two pulse sequences had been tested. The first

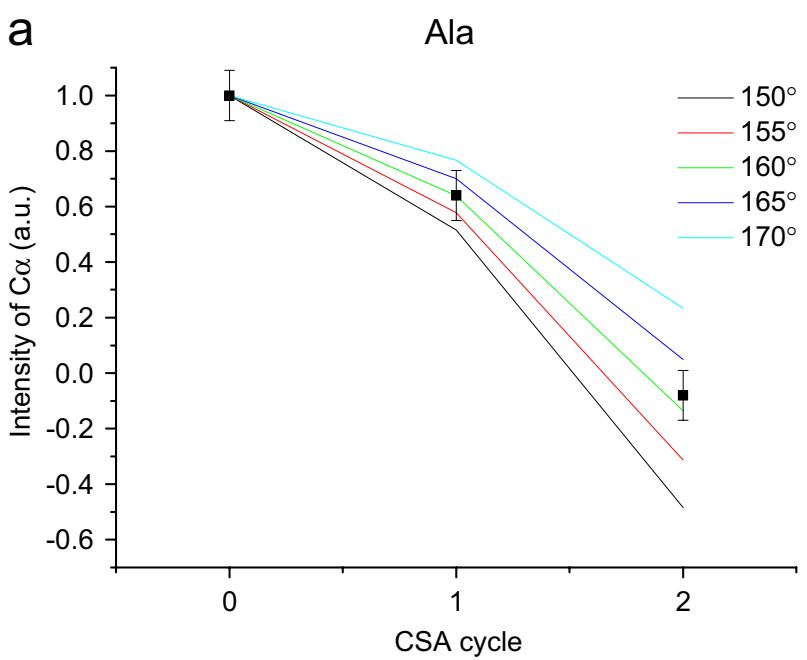

b

b

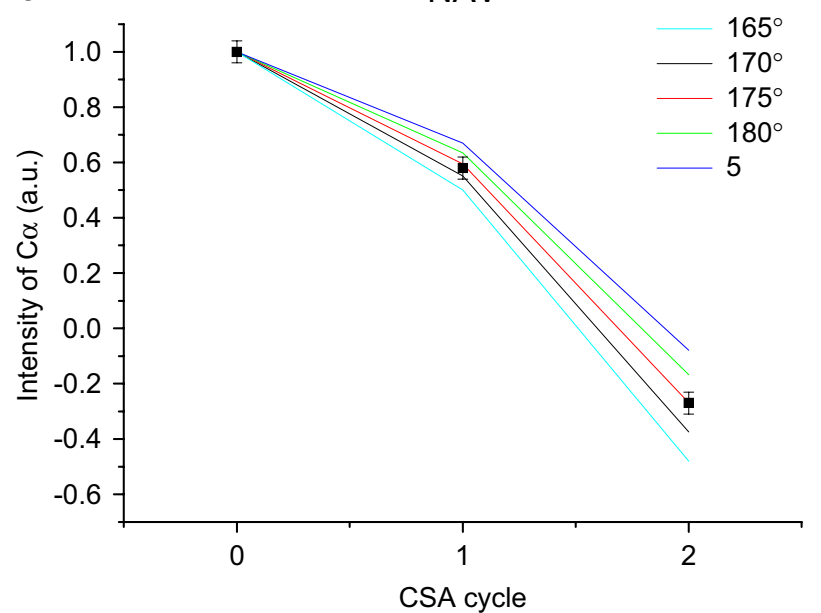

C

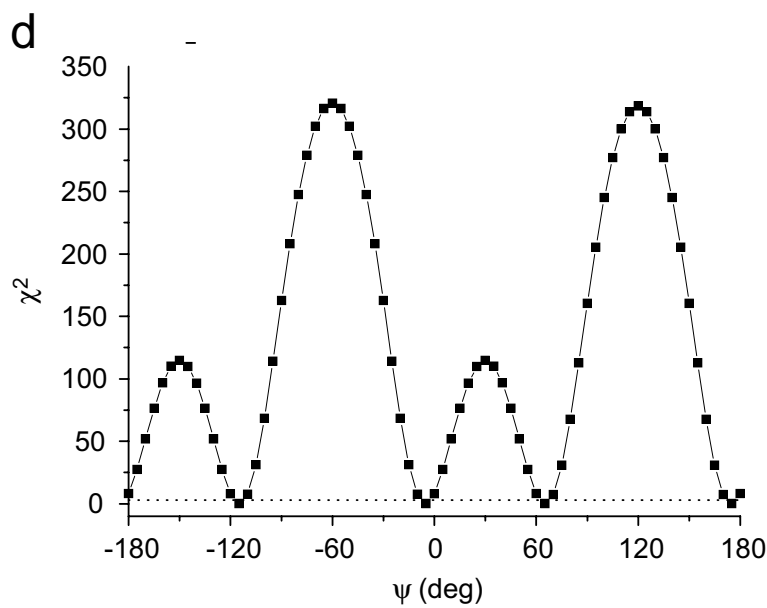

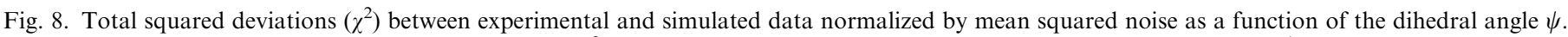

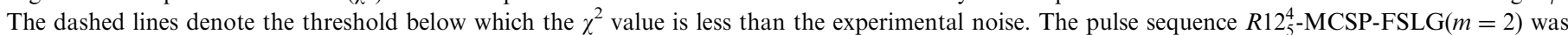
used in the measurements. 
one is known as the rotary resonance, which is $\gamma$ encoded [43]. The second one is the non- $\gamma$-encoded ${ }^{13} \mathrm{C}-{ }^{1} \mathrm{H}$ dipolar recoupling sequence based on the frequency-switched LG (FSLG) irradiations [44,45], where the spatial part of the average Hamiltonian during the dipolar recoupling period can be tailored to contain the term $\bar{\omega}_{m=2}^{D_{\mathrm{IS}}} \cos 2 \gamma$ by a judicious choice of the synchronization condition. Note that the term $\cos 2 \gamma$ is appended for the non$\gamma$-encoded Hamiltonian. We carried out a series of simulations by solving the Liouville-von Neumann equation numerically on the basis of our pulse sequence shown in Fig. 6. Our calculations were done on a three-spin $\left(\mathrm{C}^{\prime}, \mathrm{C}_{\alpha}\right.$ and $\left.\mathrm{H}_{\alpha}\right)$ systems at $7.05 T$ and at an MAS frequency of $25 \mathrm{kHz}$. The initial density operator was set as the $S_{z}$ operator of $\mathrm{C}^{\prime}$. The $\mathrm{C}_{\alpha}-\mathrm{H}_{\alpha}$ dipolar recoupling period was fixed at three rotor periods. The signal of $\mathrm{C}_{\alpha}$ was monitored as a function of the CSA recoupling period and the angle $\psi$. Referring to Fig. 7(a), the maximum span of the $\mathrm{C}_{\alpha}$ signal subtended by the interval of $\psi$ amounts to $40 \%$ of the initial $\mathrm{C}^{\prime}$ intensity, which is consistent with our simulation results based on the analytical equations (Fig. 5). Fig. 7(b) shows the corresponding results for the FSLG dipolar recoupling sequence which is non- $\gamma$ encoded. The maximum span of the $\mathrm{C}_{\alpha}$ signal is diminished to about $30 \%$ of the initial $\mathrm{C}^{\prime}$ intensity. Nevertheless, the FSLG pulse sequence has the advantages that it is well compensated for rf inhomogeneity and that the homonuclear dipole-dipole interaction is largely suppressed [45]. Therefore, $R 12_{5}^{4}$-MCSP-FSLG $(m=2)$ can be considered as a good experimental approach for $\psi$ angle determination, in which the terms $\bar{\omega}_{m=2}^{\mathrm{CS}}$ and $\bar{\omega}_{m=2}^{D_{\text {IS }}} \cos 2 \gamma$ are recoupled. a

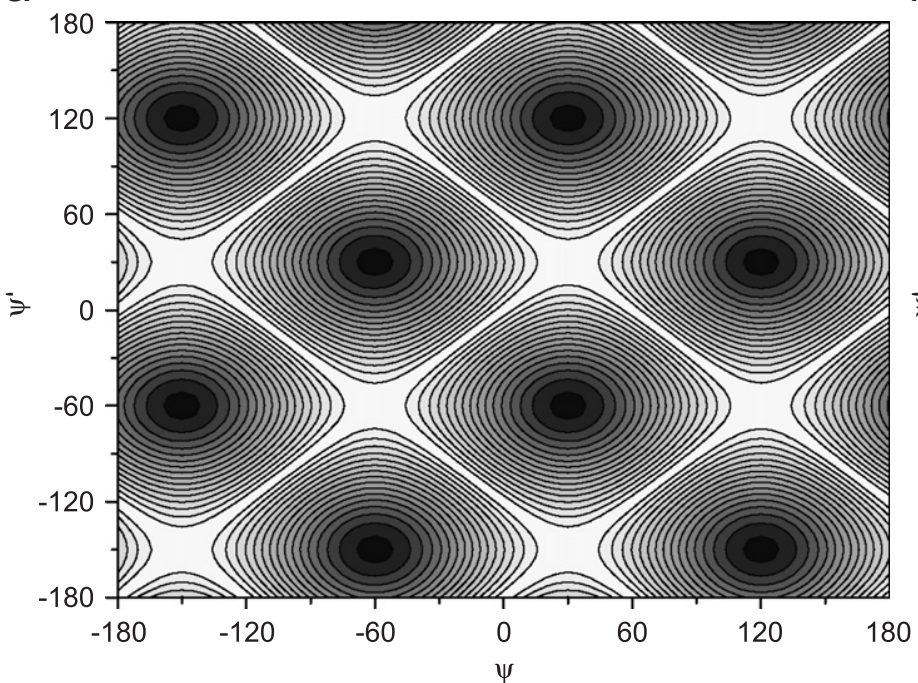

b

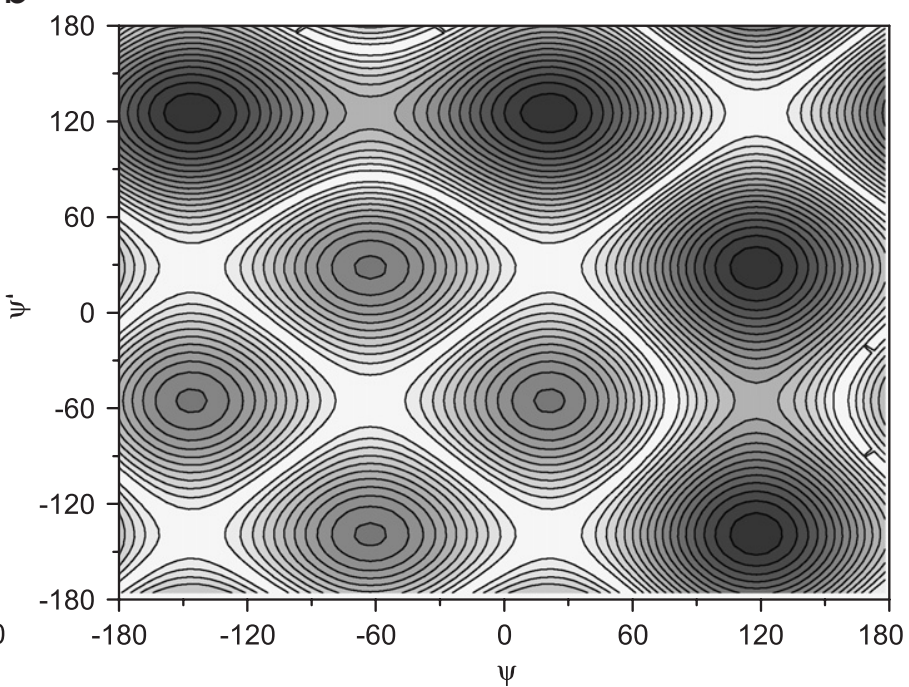

C

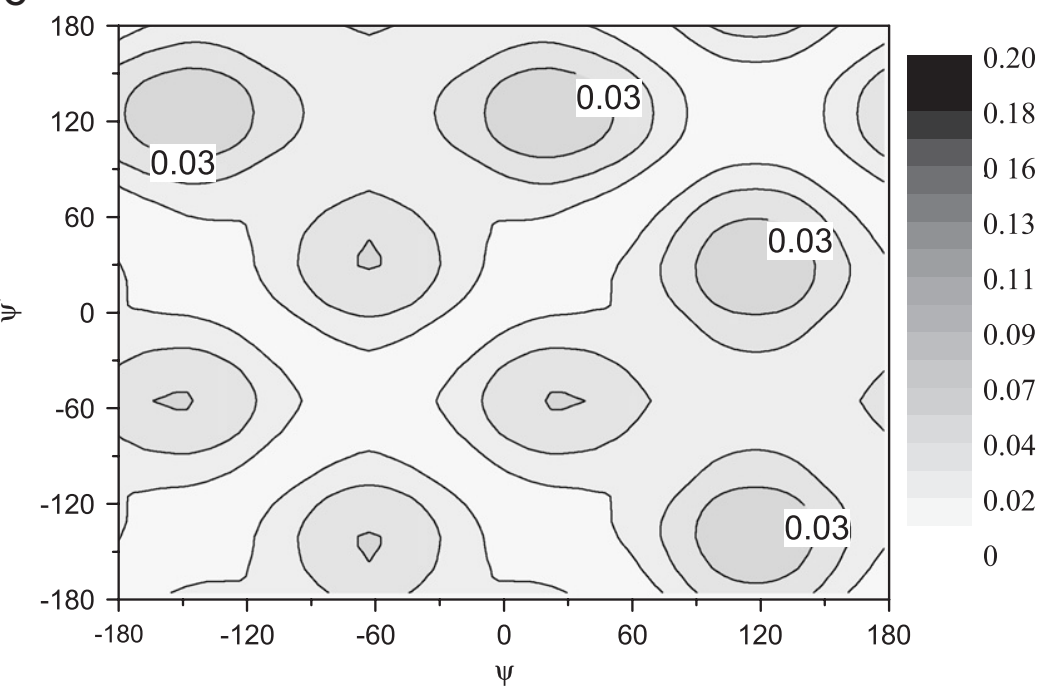

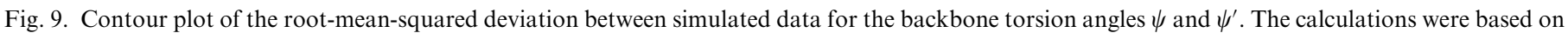

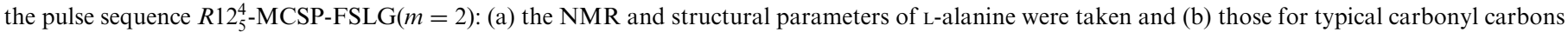

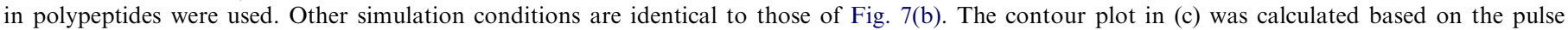
sequence ROCSA-LG. 


\subsection{Experiments on model compounds}

The experimental strategy discussed above was applied to determine the dihedral angle (NCCO) of uniformly ${ }^{13} \mathrm{C}$ and ${ }^{15} \mathrm{~N}$ labeled L-alanine and NAV samples. The ${ }^{13} \mathrm{C}^{\prime} \rightarrow \mathrm{C}_{\alpha}$ peak intensities of three separate experiments corresponding to 0,1 and 2 complete cycles of $R 12_{5}^{4}$ were measured while the dipolar recoupling period was fixed at three rotor periods. The experimental data were normalized by the first data point (no CSA recoupling) and then compared with the numerical simulations carried out in the same fashion as discussed earlier. All the simulation parameters were matched to the experimental conditions. Fig. 8 shows the experimental results and the corresponding mean-squaredeviation $\left(\chi^{2}\right)$ between experiments and simulations as a function of the $\psi$ value assumed in the simulations. Reflection symmetries about $-150^{\circ},-60^{\circ}, 30^{\circ}$ and $120^{\circ}$ are due to the symmetry properties of the $\mathrm{C}^{\prime} \mathrm{CSA}$ and the $\mathrm{C}_{\alpha}-\mathrm{H}_{\alpha}$ dipolar interactions [17]. Minimum $\chi^{2}$ values for L-alanine and NAV occur at $\psi=160^{\circ}\left(161.5^{\circ}\right)$ and $175^{\circ}$ $\left(178.2^{\circ}\right)$, respectively, where the bracketed values correspond to the crystallographic data obtained by diffraction techniques [26,42]. Comparable results were obtained when the pulse sequence was extended to its $2 \mathrm{D}$ version, where the signals in the $\mathrm{C}^{\prime}-\mathrm{C}_{\alpha}$ cross-peak region were analyzed.

\section{Discussion and conclusion}

The feasibility of determining the backbone torsion $\psi$ angle by correlating the carbonyl CSA and the $\mathrm{C}_{\alpha}-\mathrm{H}_{\alpha}$ dipolar tensors has been demonstrated experimentally in two earlier studies $[17,18]$. In this work, we find that the RACO strategy is optimized if the orientation dependence of the average Hamiltonians during the CSA and dipolar recoupling periods are in the closest resemblance. An immediate consequence is that the homonuclear polarization transfer in RACO must be isotropic to avoid any deterioration of the correlation. Furthermore, our analytical analysis shows that the optimized option is to select the average Hamiltonians with the spatial parts corresponding to $\bar{\omega}_{m=2}^{\mathrm{CS}}$ and $\bar{\omega}_{m=2}^{D_{\mathrm{IS}}}$ for the CSA and dipolar recoupling periods, respectively. We have validated our finding by experimental measurements on two model crystalline compounds under fast MAS condition. The dihedral angles determined are in excellent agreement with the diffraction data. It is remarkable that only three data points are required for an accurate determination of the dihedral angle.

While the experimental results are very encouraging for the model compounds, there are some inherent limitations in the RACO approach. Because of the symmetry properties of the CSA and dipolar interactions, it is impossible to obtain a unique determination of the $\psi$ angle by RACO alone. To clarify further the range of applicability, we show in Fig. 9(a,b) the simulated contour plots demonstrating the sensitivity of our approach over the full range of $\psi$ values, where the principal values and orientations of the
CSA tensor of L-alanine and those for typical carbonyl carbons in polypeptides were used. The contour plot for L-alanine has four reflection symmetries about the positions of $\psi=-150,-60^{\circ}, 30^{\circ}$ and $120^{\circ}$ because the orientation of the $\delta_{11}$ component of the $\mathrm{C}^{\prime}$ CSA tensor bisects the angle of $\mathrm{O}-\mathrm{C}-\mathrm{O}$. For typical polypeptides, the reflection symmetries at $\psi=-150$ and $30^{\circ}$ are lifted due to the slightly different orientation of the $\mathrm{C}^{\prime}$ CSA tensors. In our previous communication, we showed that ROCSA [25] and LG doubling [46] can be used for the CSA and heteronuclear dipolar recoupling modules of RACO for $\psi$ angle determination [18]. This ROCSA-LG approach is not optimized for RACO-like experiments because the $\mathrm{C}^{\prime} \rightarrow \mathrm{C}_{\alpha}$ polarization transfer is not isotropic and the LG irradiation selects the spatial part of $m=1$. As expected, the overall sensitivity of ROCSA-LG for $\psi$ angle determination is inferior to $R 12_{5}^{4}$-MCSP-FSLG $(m=2)$.

\section{Acknowledgments}

This work was supported by grants from the National Science Council. We thank the constructive comments of the anonymous reviewers.

\section{References}

[1] M. Baldus, Prog. Nucl. Magn. Reson. Spectrosc. 41 (2002) 1-47.

[2] R. Tycko, Prog. Nucl. Magn. Reson. Spectrosc. 42 (2003) 53-68.

[3] R. Tycko, Curr. Opin. Struct. Biol. 14 (2004) 96-103.

[4] X. Feng, Y.K. Lee, D. Sandstrom, M. Eden, H. Maisel, A. Sebald, M.H. Levitt, Chem. Phys. Lett. 257 (1996) 314-320.

[5] P.M. Henrichs, M. Linder, J. Magn. Reson. 58 (1985) 458.

[6] P. Robyr, B.H. Meier, P. Fischer, R.R. Ernst, J. Am. Chem. Soc. 116 (1994) 5315.

[7] K. Schmidt-Rohr, J. Am. Chem. Soc. 118 (1996) 7601-7603.

[8] R. Tycko, G. Dabbagh, J. Am. Chem. Soc. 113 (1991) 3592-3593.

[9] F.J. Blanco, R. Tycko, J. Magn. Reson. 149 (2001) 131-138.

[10] P.V. Bower, N. Oyler, M.A. Mehta, J.R. Long, P.S. Stayton, G.P. Drobny, J. Am. Chem. Soc. 121 (1999) 8373-8375.

[11] P.R. Costa, J.D. Gross, M. Hong, R.G. Griffin, Chem. Phys. Lett. 280 (1997) 95-103.

[12] M. Eden, A. Brinkmann, H. Luthman, L. Eriksson, M.H. Levitt, J. Magn. Reson. 144 (2000) 266-279.

[13] X. Feng, M. Eden, A. Brinkmann, H. Luthman, L. Eriksson, A. Graslund, O.N. Antzutkin, M.H. Levitt, J. Am. Chem. Soc. 119 (1997) 12006-12007.

[14] V. Ladizhansky, M. Veshtort, R.G. Griffin, J. Magn. Reson. 154 (2002) 317-324.

[15] B. Reif, M. Hohwy, C.P. Jaroniec, C.M. Rienstra, R.G. Griffin, J. Magn. Reson. 145 (2000) 132-141.

[16] C.M. Rienstra, M. Hohwy, L.J. Mueller, C.P. Jaroniec, B. Reif, R.G. Griffin, J. Am. Chem. Soc. 124 (2002) 11908-11922.

[17] Y. Ishii, T. Terao, M. Kainosho, Chem. Phys. Lett. 256 (1996) 133-140.

[18] J.C.C. Chan, R. Tycko, J. Am. Chem. Soc. 125 (2003) 11828-11829.

[19] I. Schnell, Prog. Nucl. Magn. Reson. Spectrosc. 45 (2004) 145-207.

[20] M.H. Levitt, Symmetry-based pulse sequence in magic-angle spinning solid-state NMR, in: D.M. Grant, R.K. Harris (Eds.), Encyclopedia in Nuclear Magnetic Resonance, vol. 9, Wiley, Chichester, 2002, pp. 165-196.

[21] U. Haeberlen, High resolution NMR in solids: selective averaging, in: J.S. Waugh (Ed.), Advances in Magnetic Resonance, Supplement 1, Academic Press, New York, 1976. 
[22] M. Mehring, High Resolution NMR Spectroscopy in Solids, Springer, Berlin, 1983.

[23] Y. Nishiyama, T. Yamazaki, T. Terao, J. Chem. Phys. 124 (2006).

[24] Y. Nishiyama, A. Kubo, T. Terao, J. Chem. Phys. 119 (2003) 3297-3308.

[25] J.C.C. Chan, R. Tycko, J. Chem. Phys. 118 (2003) 8378-8389.

[26] M.S. Lehmann, T.F. Koetzle, W.C. Hamilton, J. Am. Chem. Soc. 94 (1972) 2657-2660.

[27] A. Naito, S. Ganapathy, K. Akasaka, C.A. McDowell, J. Chem. Phys. 74 (1981) 3190-3197.

[28] M. Bak, N.C. Nielsen, J. Magn. Reson. 125 (1997) 132-139.

[29] M. Baldus, R.J. Iuliucci, B.H. Meier, J. Am. Chem. Soc. 119 (1997) $1121-1124$.

[30] M. Baldus, B.H. Meier, J. Magn. Reson. A 121 (1996) 65-69.

[31] J.C.C. Chan, G. Brunklaus, Chem. Phys. Lett. 349 (2001) 104-112.

[32] E.H. Hardy, A. Detken, B.H. Meier, J. Magn. Reson. 165 (2003) 208-218.

[33] E.H. Hardy, R. Verel, B.H. Meier, J. Magn. Reson. 148 (2001) 459-464.

[34] A.S.D. Heindrichs, H. Geen, C. Giordani, J.J. Titman, Chem. Phys. Lett. 335 (2001) 89-96.
[35] Y. Mou, J.C.H. Chao, J.C.C. Chan, Solid State Nucl. Magn. Reson. 29 (2006) 278-282.

[36] Y. Mou, J.C.C. Chan, Chem. Phys. Lett. 419 (2006) 144-148.

[37] S. Hediger, B.H. Meier, R.R. Ernst, Chem. Phys. Lett. 240 (1995) $449-456$.

[38] S. Hediger, B.H. Meier, N.D. Kurur, G. Bodenhausen, R.R. Ernst, Chem. Phys. Lett. 223 (1994) 283-288.

[39] A. Detken, E.H. Hardy, M. Ernst, B.H. Meier, Chem. Phys. Lett. 356 (2002) 298-304.

[40] M. Lee, W.I. Goldburg, Phys. Rev. A 140 (1965) 1261.

[41] M. Bak, J.T. Rasmussen, N.C. Nielsen, J. Magn. Reson. 147 (2000) 296-330.

[42] P.J. Carroll, P.L. Stewart, S.J. Opella, Acta Crystallogr. C-Cryst. Struct. Commun. 46 (1990) 243-246.

[43] M.H. Levitt, T.G. Oas, R.G. Griffin, Isr. J. Chem. 28 (1988) 271-282.

[44] K. Takegoshi, T. Terao, Solid State Nucl. Magn. Reson. 13 (1999) 203-212.

[45] A. Bielecki, A.C. Kolbert, H.J.M. de Groot, R.G. Griffin, M.H. Levitt, Adv. Magn. Reson. 14 (1990) 111-124.

[46] M. Hong, J.D. Gross, C.M. Rienstra, R.G. Griffin, K.K. Kumashiro, K. Schmidt-Rohr, J. Magn. Reson. 129 (1997) 85-92. 